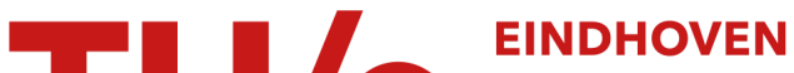 \\ UNIVERSITY OF \\ TECHNOLOGY
}

\section{On the formulation of the dynamic mixed subgrid-scale model}

Citation for published version (APA):

Vreman, A. W., Geurts, B. J., \& Kuerten, J. G. M. (1994). On the formulation of the dynamic mixed subgrid-scale model. Physics of Fluids, 6(12), 4057-4059. https://doi.org/10.1063/1.868333

DOI:

10.1063/1.868333

Document status and date:

Published: 01/01/1994

\section{Document Version:}

Publisher's PDF, also known as Version of Record (includes final page, issue and volume numbers)

\section{Please check the document version of this publication:}

- A submitted manuscript is the version of the article upon submission and before peer-review. There can be important differences between the submitted version and the official published version of record. People interested in the research are advised to contact the author for the final version of the publication, or visit the $\mathrm{DOI}$ to the publisher's website.

- The final author version and the galley proof are versions of the publication after peer review.

- The final published version features the final layout of the paper including the volume, issue and page numbers.

Link to publication

\section{General rights}

Copyright and moral rights for the publications made accessible in the public portal are retained by the authors and/or other copyright owners and it is a condition of accessing publications that users recognise and abide by the legal requirements associated with these rights.

- Users may download and print one copy of any publication from the public portal for the purpose of private study or research.

- You may not further distribute the material or use it for any profit-making activity or commercial gain

- You may freely distribute the URL identifying the publication in the public portal.

If the publication is distributed under the terms of Article $25 \mathrm{fa}$ of the Dutch Copyright Act, indicated by the "Taverne" license above, please follow below link for the End User Agreement:

www.tue.nl/taverne

Take down policy

If you believe that this document breaches copyright please contact us at:

openaccess@tue.nl

providing details and we will investigate your claim. 


\title{
On the formulation of the dynamic mixed subgrid-scale model
}

\author{
Bert Vreman, Bernard Geurts, and Hans Kuerten \\ Department of Applied Mathematics, University of Twente, P.O. Box 217, 7500 AE Enschede, \\ The Netherlands
}

(Received 24 February 1994; accepted 27 July 1994)

\begin{abstract}
The dynamic mixed subgrid-scale model of Zang et al. [Phys. Fluids A 5, 3186 (1993)] (DMM1) is modified with respect to the incorporation of the similarity model in order to remove a mathematical inconsistency. Compared to DMM1, the magnitude of the dynamic model coefficient of the modified model (DMM2) is increased considerably, while it is still significantly smaller than as occurs in the dynamic subgrid-scale eddy-viscosity model of Germano [J. Fluid Mech. 238, 325 (1992)] (DSM). Large eddy simulations (LES) for the weakly compressible mixing layer are conducted using these three models and results are compared with direct numerical simulation (DNS) data. LES based on DMM1 gives a significant improvement over LES using DSM, while even better agreement is achieved with DMM2. (C) 1994 American Institute of Physics.
\end{abstract}

The occurrence of small scale structures in turbulent flows prevents a direct numerical simulation (DNS) of the Navier-Stokes equations, even in simple geometries. Therefore, much attention is paid to large eddy simulation (LES), in which the large scales are solved explicitly, while the effect of the small (subgrid) scales is modeled with a subgridscale model. ${ }^{1}$ The most widely used subgrid-scale model is the Smagorinsky eddy-viscosity model. ${ }^{2}$ In order to overcome certain drawbacks of the Smagorinsky model, Germano $^{3}$ proposed a dynamic procedure for the model coefficient. This dynamic subgrid-scale eddy-viscosity model (DSM) has been applied successfully to a variety of flows (e.g., Refs. 4-6). Recently, Zang et al. ${ }^{7}$ formulated a dynamic mixed model (DMM1), which employs the dynamic procedure on the mixed model of Bardina et al. ${ }^{8}$ This model does not require the assumption that the principal axes of the turbulent stress tensor are aligned with those of the strain rate tensor. The results obtained with DMM1 were observed to be more accurate when compared to those obtained with DSM for the driven cavity. In this paper an alternative, mathematically consistent formulation for the dynamic mixed model (DMM2) is proposed. Furthermore, we compare results of DMM2 with those of DSM and DMM1, using LES for the three-dimensional weakly compressible mixing layer.

We focus on the modeling of the turbulent stress tensor and for sake of transparency we present the incompressible formulation. The first step in the LES-approach consists of filtering a flow variable, e.g., the velocity component $u_{i}$, as follows:

$$
\bar{u}_{i}(\mathbf{x}, t)=\int \bar{G}(\mathbf{x}-\mathbf{z}) u_{i}(\mathbf{z}, t) d \mathbf{z},
$$

where $\bar{G}$ is a filter function with filter width $\bar{\Delta}$, defining the filter on the " $\bar{G}$ level." If this filter operation is applied to the Navier-Stokes equations, subgrid-terms appear, which are expressed in the turbulent stress tensor

$$
\tau_{i j}=\overline{u_{i} u_{j}}-\bar{u}_{i} \bar{u}_{j} \text {. }
$$

This tensor has to be modeled in terms of the filtered velocities $\bar{u}_{i}$ in order to close the equations. Germano introduced another filter, the explicit test filter on the " $\hat{G}$ level" with filter width $\hat{\Delta}$. Furthermore, the consecutive application of $\tilde{G}$ and $\hat{G}$ to a signal $\left(u_{i} \rightarrow \hat{u}_{i}\right)$ defines the filter function $\hat{G}$ (which is the convolution of $\bar{G}$ and $\hat{G}$ ) with filter width $\hat{\Delta}$. The turbulent stress on the $\hat{G}$ level is defined as

$$
T_{i j}=\widehat{\overline{u_{i} u_{j}}}-\hat{u}_{i} \hat{u}_{j} \text {. }
$$

Moreover, the following algebraic relation between the turbulent stresses on the two filter-levels was derived:

$$
T_{i j}-\hat{\tau}_{i j}=L_{i j},
$$

where

$$
L_{i j}=\widehat{\vec{u}_{i} \bar{u}_{j}}-\hat{\hat{u}}_{i} \hat{\vec{u}}_{j}
$$

is the resolved turbulent stress. This "Germano" identity has been used to dynamically obtain model coefficients which appear in the formulation of subgrid models.

The first model which has been substituted into the identity is the Smagorinsky eddy-viscosity model, which reads

$$
\tau_{i j}^{a}=-2 c_{S} \bar{\Delta}^{2}|\bar{S}| \bar{S}_{i j},
$$

where

$$
\begin{aligned}
& \bar{S}_{i j}=\frac{1}{2}\left(\frac{\partial \bar{u}_{i}}{\partial x_{j}}+\frac{\partial \bar{u}_{j}}{\partial x_{i}}\right), \\
& |\bar{S}|=\left(2 \bar{S}_{i j} \bar{S}_{i j}\right)^{1 / 2} .
\end{aligned}
$$

The model coefficient $c_{S}$ is allowed to be a function of space and time. Furthermore, in Eq. (6) and in the following the superscript " $a$ " denotes the anisotropic part of the tensor. On the $\stackrel{G}{G}$ level the model reads

$$
T_{i j}^{a}=-2 c_{S} \hat{\Delta}^{2}|\hat{S}| \hat{S}_{i j},
$$

where $\hat{S}_{i j}$ and $|\hat{S}|$ are defined by analogy with Eqs. (7) and (8). Substituting (6) and (9) into the anisotropic part of identity (4) yields

$$
c_{S} M_{i j}=L_{i j}^{a},
$$

with

$$
M_{i j}=-2 \hat{\Delta}^{2}|\hat{S}| \hat{S}_{i j}+2 \bar{\Delta}^{2} \mid{\widehat{\hat{S}} \mid \bar{S}_{i j}}
$$


To obtain the expression for $M_{i j}$ we have neglected the variation of $c_{S}$ on the scale of the test filter width $\hat{\Delta}$. Since Eq. (10) represents a system of equations for the single unknown $c_{S}$, a least-square approach ${ }^{9}$ is used to calculate this coefficient:

$$
c_{S}=\frac{\left\langle M_{i j} L_{i j}^{a}\right\rangle}{\left\langle M_{i j} M_{i j}\right\rangle} .
$$

The brackets $\langle\cdot\rangle$ denote an average over the homogeneous directions which is introduced additionally in order to stabilize actual calculations with DSM. More sophisticated procedures for the determination of $c_{S}$ have been proposed (see Ref. 10 for a survey).

Rather than starting from the Smagorinsky model Zang et $a l^{7}$ have adopted the mixed model as base model:

$$
\tau_{i j}^{a}=\left(\overline{\bar{u}_{i} \overline{\bar{u}} \bar{u}_{j}}-\overline{\bar{u}}_{i} \overline{\bar{u}}_{j}\right)^{a}-2 c_{S} \bar{\Delta}^{2}|\bar{S}| \bar{S}_{i j} .
$$

The first term on the right-hand side is the similarity model, whereas the second part represents the model for the unresolved residual stress, adopting the Smagorinsky eddyviscosity formulation. Next, identity (4) is used to obtain the model coefficient $c_{S}$. Zang et al. ${ }^{7}$ write the turbulent stress on the $\hat{\bar{G}}$ level as

$$
T_{i j}^{a}=\left(\widehat{\overline{\bar{u}}_{i} \bar{u}_{j}}-\hat{\tilde{u}}_{i} \hat{\bar{u}}_{j}\right)^{a}-2 c_{S} \hat{\Delta}^{2}|\hat{S}| \hat{S}_{i j} .
$$

Substituting (13) and (14) into the Germano identity for the anisotropic part yields

$$
H_{i j}^{a}+c_{S} M_{i j}=L_{i j}^{a},
$$

with $M_{i j}$ and $L_{i j}$ given by (11) and (5), respectively. The tensor $H_{i j}$ is defined as

$$
H_{i j}=\widehat{\overline{\bar{u}}_{i} \bar{u}_{j}}-\hat{\hat{\bar{u}}_{i}} \widehat{\overline{\bar{u}}_{j}}-\left(\widehat{\overline{\bar{u}_{i} \overline{\bar{u}}_{j}}}-\widehat{\overline{\bar{u}}_{i} \overline{\bar{u}}_{j}}\right)=\widehat{\overline{\bar{u}}_{i} \bar{u}_{j}}-\widehat{\overline{\tilde{u}}_{i}} \widehat{\overline{\bar{u}}_{j}} \text {. }
$$

Finally, $c_{S}$ is determined by analogy with Eq. (12),

$$
c_{S}=\frac{\left\langle M_{i j}\left(L_{i j}-H_{i j}\right)\right\rangle}{\left\langle M_{i j} M_{i j}\right\rangle}
$$

which completes the formulation of the dynamic mixed model (DMM1).

In order to arrive at the alternative formulation (DMM2), it is essential to observe the inconsistency resulting from the use of the $\bar{G}$ level filtered velocity in the model for $T_{i j}$ in Eq. (14). The tensor $\tau_{i j}$ is the turbulent stress on the $\bar{G}$ level and its model is expressed in the $\bar{G}$-filtered velocity $\left(\tilde{u}_{i}\right)$ only, according to Eq. (13). In order to be mathematically consis-

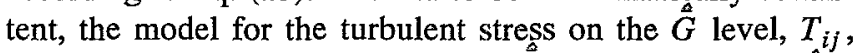
should entirely be expressed in the $\hat{G}$-filtered velocity $\left(\hat{u}_{i}\right)$. However, in Eq. (14), the similarity part depends on $\bar{u}_{i}$, while the eddy-viscosity part depends on $\hat{\vec{u}}_{i}$. Therefore, we propose to replace Eq. (14) by the following expression, in which both the similarity and the eddy-viscosity part are expressed in terms of $\hat{\bar{u}}_{i}$ :

$$
T_{i j}^{a}=\left(\widehat{\hat{\bar{u}}_{i} \vec{u}_{j}}-\hat{\hat{\hat{u}}} \hat{\vec{\Delta}}_{j}\right)^{a}-2 c_{S} \hat{\Delta}^{2}|\hat{S}| \hat{\hat{S}_{i j}} \text {. }
$$

Thus we obtain instead of (16) the following $H_{i j}$ tensor:

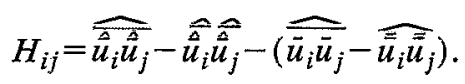

With this expression and the previously introduced $M_{i j}$ and $L_{i j}$, the model coefficient $c_{S}$ is obtained using Eq. (17), which completes the alternative formulation of the dynamic mixed model (DMM2).

In the following, we compare results of LES using the three different dynamic subgrid models described above. As an example, we consider the temporal, weakly compressible mixing layer in a cubic domain. The length of the domain is equal to four times the wavelength of the most unstable mode provided by linear stability theory. The scenario of this flow shows the roll-up of the spanwise vorticity, resulting in four spanwise rollers at the nondimensional time $t=20$. Subsequently, pairing of these rollers is observed, reducing the number of rollers to two at $t=40$. The final pairing is accomplished at $t=80$, at which time the complicated structure of the flow is highly three dimensional.

Large eddy simulations are conducted up to $t=100$, solving the compressible Navier-Stokes equations at a low Mach number of 0.2 . It has been verified that compressibility effects affecting the subgrid-modeling are very small for this flow at the current Mach number ${ }^{11}$ and, hence, only a subgrid-model for the anisotropic part of the turbulent stress tensor needs to be adopted. The spatial discretization is fourth-order accurate for the convective and second-order accurate for the viscous terms. The collocated grid contains $32^{3}$ cells of size $h$. The box filter is adopted with $\bar{\Delta}=2 h$, while the convolution integral is calculated with the trapezoidal rule. The filter width of the test filter is chosen to be twice as large, i.e., $\hat{\Delta}=2 \bar{\Delta}$, whereas the filter width on the $\hat{G}$ level is obtained using

$$
\hat{\Delta}^{2}=\bar{\Delta}^{2}+\hat{\Delta}^{2} \text {. }
$$

This relation is exact for Gaussian filters. ${ }^{3}$ For box filters a difficulty arises, since the consecutive application of two box filters is not a box filter, but a "trapezoid" filter. This trapezoid filter function $\hat{\vec{G}}$ is optimally approximated by a box

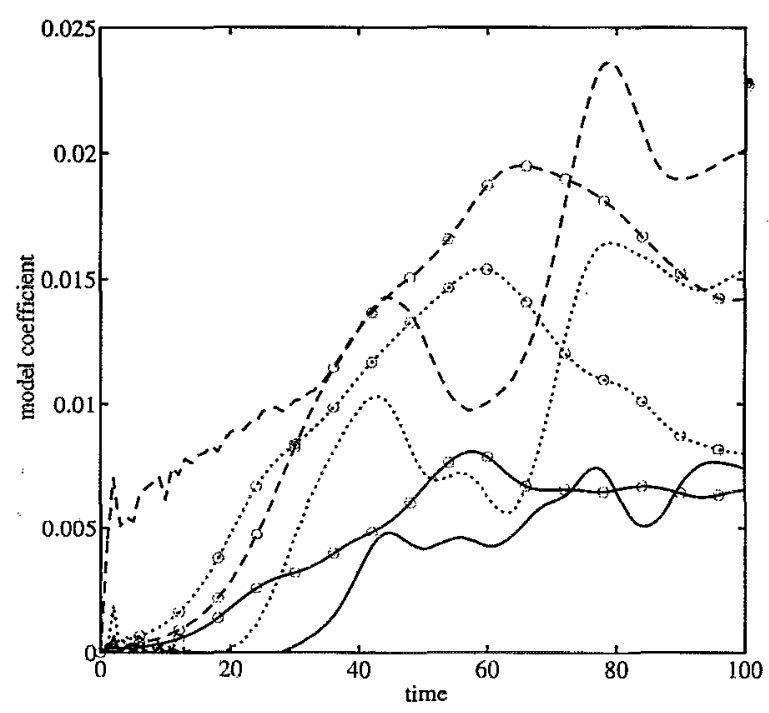

FIG. 1. The coefficient $c_{S}$ for LES with DSM (dashed), DMM1 (solid) and DMM2 (dotted) at two locations: $x_{2}=-14.75$ (marker "O") and $x_{2}=0$ (no marker). 


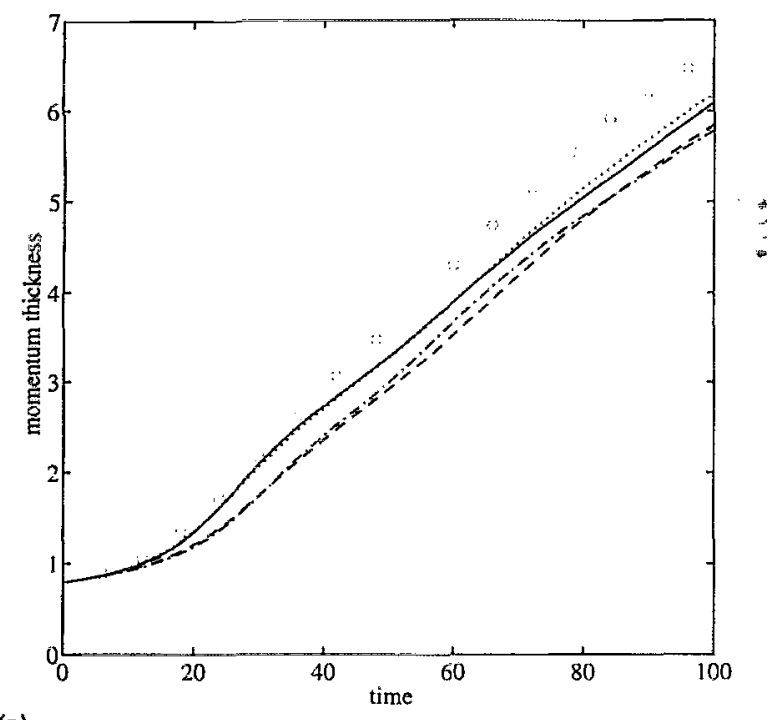

(a)

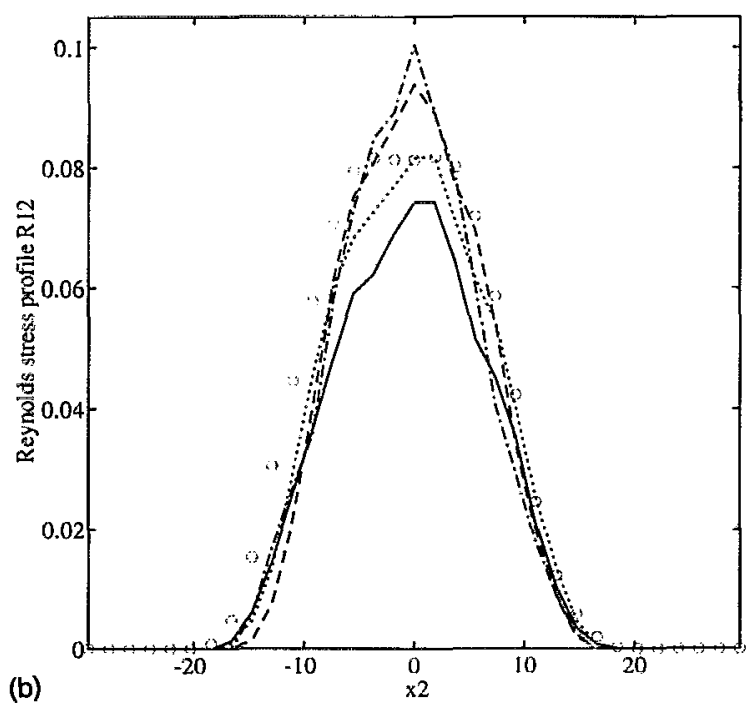

FIG. 2. The momentum thickness (a) and Reynolds stress profile $R_{12}$ at $t=70$ (b) for LES with DSM (dashed), DMM1 (solid) and DMM2 (dotted) compared with a coarse-grid DNS (dashed-dotted) and the filtered fine-grid DNS (marker "O").

filter (say $F$ ) with filter width $\hat{\Delta}$. The $L_{2}$ norm of $\hat{\bar{G}}-F$ attains the minimum value when relation $(20)$ is satisfied. Actual integrations over a volume of size $\Delta$ are not performed; the $\hat{G}$ filter is applied by the consecutive integrations over volumes with size $\bar{\Delta}$ and $\hat{\Delta}$, respectively. Relation (20) is only used for the calculation of the first term in $M_{i j}$ [Eq. (11)]. In order to perform the filtering numerically, averaging procedures similar to those described in Appendix A of Ref. 7 are used.

Figure 1 shows the value of $c_{S}$ for DSM, DMM1, and DMM2, respectively, obtained from large eddy simulations using these models. The coefficient $c_{S}$ is obtained using formula (12) for DSM and (17) for DMM1 and DMM2. Averaging over the two homogeneous directions renders the coefficient $c_{S}$ as a function of time and the normal direction $x_{2}$.
For all three models, $c_{S}$ appears to become negative only in very small parts of the flow. In Fig. 1 the evolution of $c_{S}$ is shown for two values of $x_{2}$. As expected, DSM is observed to give higher values for $c_{S}$ than the mixed models, since the eddy-viscosity part in the latter models takes only the unresolved part of the turbulent stress into account, while DSM has to model the full turbulent stress. Furthermore, DMM1 produces a substantially lower $c_{S}$ than DMM2. The reason is probably that $H_{i j}$ in Eq. (16) tends to be larger than $H_{i j}$ in Eq. (19), since the filtered velocity $\bar{u}_{i}$ contains more smallscale structures than $\hat{\vec{u}}_{i}$.

Figure 2 shows the momentum thickness and a Reynolds stress profile $R_{12}$ for LES with DSM, DMM1, and DMM2. The value of the momentum thickness also measures the spread of the mean velocity profile. Moreover, results from a filtered fine-grid DNS ( $192^{3}$ grid) and a coarse-grid DNS $\left(32^{3}\right.$ grid) are included. For all three models we observe that LES produces better results than the coarse-grid DNS at the same grid. Moreover, the dynamic mixed model DMM1 gives better agreement than DSM. The alternative formulation for the dynamic mixed model (DMM2) yields even more improvement over DSM.

Summarizing, the formulation of the recently introduced dynamic mixed model (DMM1) has been discussed and a mathematically consistent modification has been proposed (DMM2). Actual LES for the mixing layer demonstrates that this modification gives rise to higher values of the dynamic model coefficient. Furthermore, the modification improves the results, whereas both DMM1 and DMM2 are considerably better than the dynamic subgrid-scale model DSM.

\section{ACKNOWLEDGMENTS}

The authors wish to thank Professor P. Moin for a useful discussion and NWO for providing computing time through NCF.

${ }^{1}$ R. S. Rogallo and P. Moin, "Numerical simulation of turbulent flows," Annu. Rev. Fluid Mech. 16, 99 (1984).

2J. Smagorinsky, "General circulation experiments with the primitive equations. I. "The basic experiment," Mon. Weather Rev. 91, 99 (1963).

${ }^{3}$ M. Germano, "Turbulence: the filtering approach," J. Fluid Mech. 238, 325 (1992).

${ }^{4}$ M. Germano, U. Piomelli, P. Moin, and W. H. Cabot, "A dynamic subgridscale eddy viscosity model," Phys. Fluids A 3, 1760 (1991).

${ }^{5}$ P. Moin, K. Squires, W. Cabot, and S. Lee, "A dynamic subgrid-scale model for compressible turbulence and scalar transport," Phys. Fluids A 3, 2746 (1991).

${ }^{6}$ N. M. El-Hady, T. A. Zang, and U. Piomelli, "Dynamic subgrid-scale modelling for high-speed transitional boundary layers," ASME FED 162, 103 (1993).

${ }^{7}$ Y. Zang, R. L. Street, and J. R. Koseff, "A dynamic mixed subgrid-scale model and its application to turbulent recirculating flows," Phys. Fluids A 5, 3186 (1993).

${ }^{8} \mathrm{~J}$. Bardina, J. H. Ferziger, and W. C. Reynolds, "Improved turbulence models based on LES of homogeneous incompressible turbulent flows," Department of Mechanical Engineering, Report No. TF-19, Stanford, 1984.

${ }^{9} \mathrm{D}$. K. Lilly, "A proposed modification of the Germano subgrid-scale closure method," Phys. Fluids A 4, 633 (1992).

${ }^{10} \mathrm{P}$. Moin and J. Jimenez, "Large eddy simulation of complex turbulent flows," AIAA 24th Fluid Dynamics Conference, Orlando (1993).

${ }^{11}$ A. W. Vreman, B. J. Geurts, and J. G. M. Kuerten, "Subgrid-modelling in LES of compressible flows," The First ERCOFTAC Workshop on Direct and Large Eddy Simulation, Guildford (1994). 